\title{
Case series on endoscopic simultaneous multiple plastic stent dilatation of strictures following iatrogenic bile duct injury
}

\author{
Kumarasinghe KAPR* and Pathirana AA \\ Colombo South Teaching Hospital, Sri Lanka
}

\begin{abstract}
Iatrogenic bile duct injuries (IBDI) are a devastating complication of cholecystectomy. Optimal management of the IBDI depends on several factors including type of injury and availability of equipment and expertise. Reconstructive hepaticojejunostomy is a well-established and commonly performed procedure for IBDI s. However, endoscopic serial multiple stent dilation of IBDI strictures has gained popularity in the recent era. In our case series we have adopted a protocol based placement of multiple plastic stents for E2, E3 IBDI strictures with a high success rate.
\end{abstract}

\section{Background}

Bile duct injuries are a devastating complication of cholecystectomy. Since introduction of LC in 1990s, Biliary injury has doubled from $0.2 \%$ to $0.4 \%$ and remains constant despite advances in technique and technology [1]. Approximately $30 \%$ of bile duct injuries are identified during the primary surgery. Optimal management of an IBDI depends on several factors, including type of injury, associated vascular injuries, condition of the patient, timing of reconstruction and availability of expertise, and includes open surgical and endo-therapeutic options [2]. Reconstructive hepaticojejunostomy which is commonly performed, is associated with low mortality and low morbidity $[3,4]$. Minor injuries can be managed endoscopically with comparable success rates [57]. However, some reports indicate that endoscopic management is associated with increased incidence of re-stenosis, cholangitis and secondary billiary cirrhosis, Strasburg type E2 and E3 IBDI strictures are managed with serial endoscopic stenting while more severe injuries are managed with reconstructive hepatico- jejunostomy. Our practice is to place simultaneous multiple Amsterdam type stents to achieve satisfactory stricture dilatation. The stents are then kept in place for up to at least a year (with 3 monthly endoscopic assessments) and removed thereafter.

\section{Cases}

\section{Patient 1}

A 42-year-old female patient was referred to us with a bile leak after laparoscopic cholecystectomy and had a drain placed at the site of leak. She underwent ERCP on post-operative day 4 at our unit and injury type was Stratsburg- Bismuth E2 was confirmed. In the first ERCP 7F Amersterdam type plastic biliary stent inserted over a hydrophilic guidewire ( 0.035 inch in diameter). Subsequently her drain output was gradually reduced and at the end of one month it was converted to a $10 \mathrm{f}$ stent.

She underwent sequential stent insertion every 3 months and finally bile duct was stented with five $10 \mathrm{f}$ plastic stents at the end of
14 months. She was followed up with serial liver function tests in each of her clinic visits and she did not develop cholangitis throughout her management. Five $10 \mathrm{f}$ stents were kept for 3 months and then removed (Figure 1).

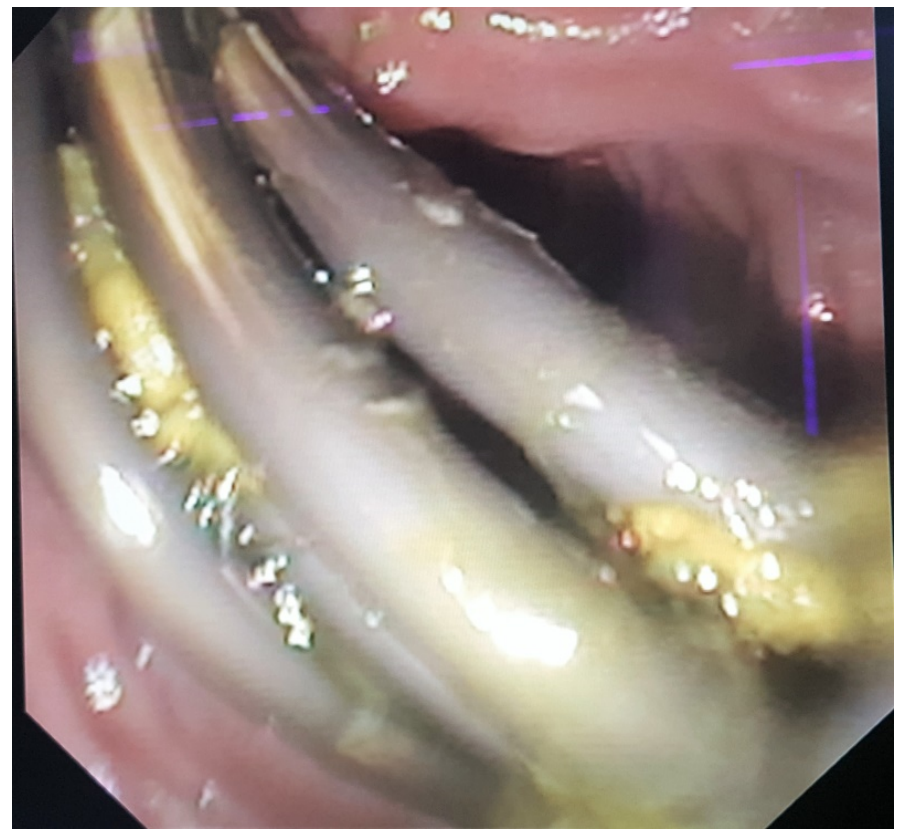

Figure 1. Five plastic stents inserted to common bile duct across the Stricture.

Correspondence to: Kumarasinghe KAPR, Colombo South Teaching Hospital, Sri Lanka, Tel: +94777914061; E-mail: prabath14@gmail.com

Key words: IBDI, hepatico-jejunostomy, endoscopic stenting

Received: December 02, 2017; Accepted: December 15, 2017; Published: December 22, 2017 


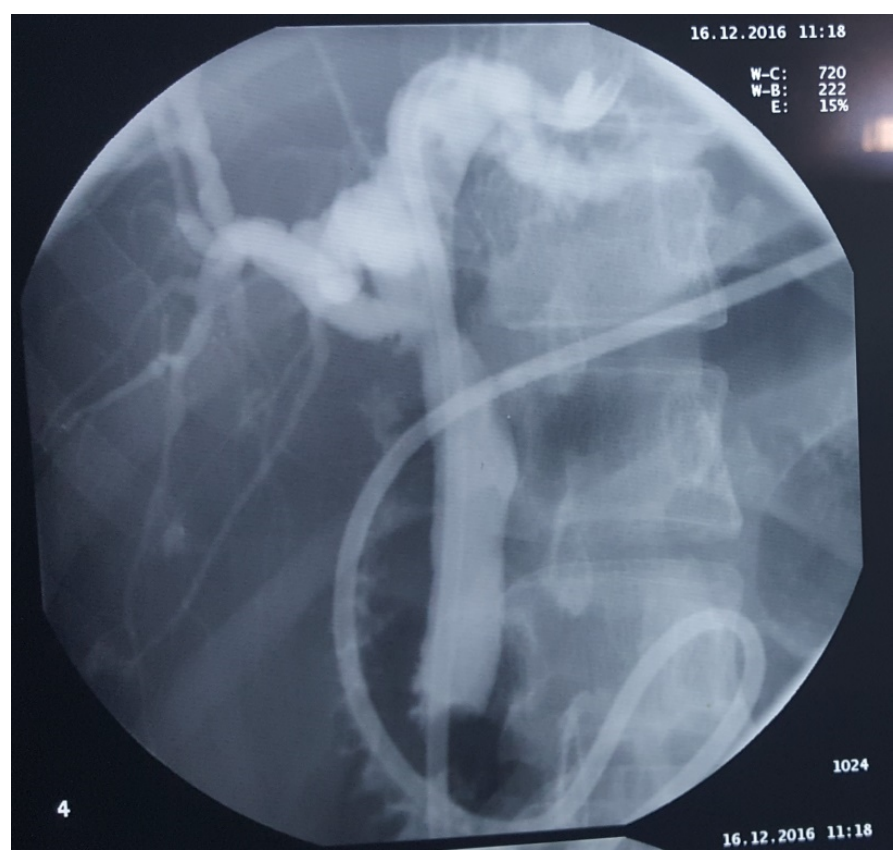

Figure 2. Cholangiogram done at 48 hours through the nasobiliary drain, after removal of stents.

Subsequent cholangiogram didn't show waist formation at the site of stricture, then a naso-billiary drain was inserted. Naso-billiary drain was kept for 48 hours and repeat cholangiogram was done, and it didn't show any evidence of re-stricture. Patient is currently on 3 monthly follow up with ultrasonography and LFT s. at the end of 6 months there is no evidence of re-stricture (Figure 2).

\section{Patient 2}

Another 48-year-old female patient was referred to us following IBDI following laparoscopic cholecystectomy. The procedure was converted to open and a drain was placed. Initial ERCP failed to cannulate the CBD as there was a "complete cut off". However, a subsequent ERCP performed a week later demonstrated a Strasburg Type E3 injury and a $7 \mathrm{f}$ plastic stent was placed across the site of stricture. She too underwent serial dilatation with $10 \mathrm{f}$ plastic stents. Ultimately $510 \mathrm{f}$ stents were placed in the CBD. In this case we used Hurricane balloon dilatation before stenting in some instances. This patient was also followed up similarly as above.

\section{Patient 3}

A 28-year-old female was referred to us with a Strasburg E2 bile duct injury following laparoscopic cholecystectomy, and she is currently on serial multiple stent dilatations with $210 \mathrm{~F}$ stents.

\section{Discussion and conclusion}

The European Society of Gastrointestinal Endoscopy (ESGE) published guidelines on endoscopic serial stenting recently [6]. According to guidelines placement of multiple plastic stents for benign strictures of common bile duct is possible in $90 \%$ of cases and patency rate following stenting is high as $90 \%$ in patients with post-operative biliary strictures [8]. Costamagna et al. [6] in his series on simultaneous multiple plastic stent placement for benign billiary strictures reported 45 completed cases with a long-term patency rate of $89 \%$, in his series mean number of ERCPS was 4 and mean number of stents used was 3.2. Duration of stenting was 12 months. At 24-48 hours a cholangiogram was done after removal of stents to confirm the patency. In another series Kuzela et al. in 2005 [5] reported 43 cases that underwent serial stenting for post cholecystectomy strictures with a long-term patency rate of $100 \%$. Mean number of ERCPs was 6 and mean number of stents was 3.4. Patients were stented up to 1year and they were followed up for 16 months post procedure. In our case series protocol, we used to placement of $7 \mathrm{~F}$ plastic stent at the 1st attempt after selective billiary sphincterotomy followed by repeat ERCP and stenting at 3month for placement of $10 \mathrm{~F}$ plastic stent. Subsequently stent number is increased every 3 monthly until adequate patency achieved. (3,4 or 5 plastic stents). Selectively hurricane balloon dilatation is used. Surgery is the best option in cases with complete transection where ERCP cannot gain access across the site of injury, whereas endoscopic simultaneous multiple stenting possible injuries like Bismuth E2, 3 with a high success rate.

\section{References}

1. Costamagna G, Boškoski I (2013) Current treatment of benign biliary strictures. Ann Gastroenterol 26: 37-40. [Crossref]

2. Archer SB, Brown DW, Smith CD, Branum GD, Hunter JG (2001) Bile duct injury during laparoscopic cholecystectomy: results of a national survey. Ann Surg 234: 549558. [Crossref]

3. Tocchi A, Costa G, Lepre L, Liotta G, Mazzoni G, et al. (1996) The long-term outcome of hepaticojejunostomy in the treatment of benign bile duct strictures. Ann Surg 224: 162-167. [Crossref]

4. Nealon WH, Urrutia F (1996) Long-term follow-up after bilioenteric anastomosis for benign bile duct stricture. Ann Surg 223: 639-645. [Crossref]

5. Kuzela L, Oltman M, Sutka J, Hrcka R, Novotna T, et al. (2005) Prospective follow-up of patients with bile duct strictures secondary to laparoscopic cholecystectomy, treated endoscopically with multiple stents. Hepatogastroenterology 2005 52: 1357-1361. [Crossref]

6. Costamagna G, Pandolfi M, Mutignani M, Spada C, Perri V (2001) Long-term results of endoscopic management of postoperative bile duct strictures with increasing numbers of stents. Gastrointest Endosc 54: 162-168. [Crossref]

7. Dumonceau JM, Devière J, Delhaye M, Baize M, Cremer M (1998) Plastic and metal stents for postoperative benign bile duct strictures: the best and the worst. Gastrointest Endosc 47: 8-17. [Crossref]

8. Dumonceau JM, Tringali A, Blero D, Devière J, Laugiers R, et al. (2012) Biliary stenting: indications, choice of stents and results: European Society of Gastrointestinal Endoscopy (ESGE) clinical guideline. Endoscopy 44: 277-298. [Crossref]

Copyright: (C2017 Kumarasinghe KAPR. This is an open-access article distributed under the terms of the Creative Commons Attribution License, which permits unrestricted use, distribution, and reproduction in any medium, provided the original author and source are credited. 